\title{
The impact of endogenous annexin A1 on glucocorticoid control of in ammatory arthritis
}

\author{
Hetal B Patel, ${ }^{1}$ Kristin N Kornerup, ${ }^{1}$ Andre LF Sampaio, ${ }^{1}$ Fulvio D Acquisto, ${ }^{1}$ Michael P \\ Seed, ${ }^{1}$ Ana Paula Girol, ${ }^{2}$ Mohini Gray, ${ }^{3}$ Costantino Pitzalis, ${ }^{1}$ Sonia M Oliani, ${ }^{2}$ Mauro \\ Perretti ${ }^{1}$
}

\begin{abstract}
- Additional ( gures and tables are published online only. To view these les please visit the journal online (http://ard.bmi.com/ content/early/recent).

'William Harvey Research Institute, Barts and The London School of Medicine, London UK 2Department of Biology; Instituto de Bioci ncias, Letras e Ci ncias Exatas (IBILCE), S o Paulo State University, S o Jos do Rio Preto, Brazil ${ }^{3}$ Medical Research Council Centre for In ammation, University of Edinburgh,

Edinburgh, UK

Experimental Medicine and

Rheumatology, William Harvey

Research Institute, London, UK
\end{abstract}

\section{Correspondence to}

Mauro Perretti, Barts and The

London School of Medicine,

William Harvey Research

Institute, Charterhouse Square, London EC1M 6BO, UK;

m.perretti@qmul.ac.uk

Accepted 23 March 2012

Published Online First

5 May 2012

\section{ABSTRACT}

Objectives To establish the role and effect of glucocorticoids and the endogenous annexin A1 (AnxA1) pathway in in ammatory arthritis.

Methods Ankle joint mRNA and protein expression of AnxA1 and its receptors were analysed in naive and arthritic mice by real-time PCR and immunohistochemistry. In ammatory arthritis was induced with the K/BxN arthritogenic serum in AnxA1+/+ and $A n \times A 1^{-1-}$ mice; in some experiments, animals were treated with dexamethasone (Dex) or with human recombinant AnxA1 or a protease-resistant mutant (termed SuperAnxA1). Readouts were arthritic score, disease incidence, paw oedema and histopathology, together with pro-in ammatory gene expression.

Results All elements of the AnxA1 pathway could be detected in naive joints, with augmentation during ongoing disease, due to the in Itration of immune cells. No difference in arthritis intensity of pro le could be observed between AnxA1 ${ }^{+/+}$and $A n x A 1^{-/-}$mice. Treatment of mice with Dex (10 $\mu \mathrm{g}$ intraperitoneally daily from day 2) afforded potent antiarthritic effects highly attenuated in the knockouts: macroscopic changes were mirrored by histopathological ndings and proin ammatory gene (eg, Nos2) expression. Presence of proteinase 3 mRNA in the arthritic joints led the authors to test AnxA1 and the mutant SuperAnxA1 (1 $\mu \mathrm{g}$ intraperitoneally daily in both cases from day 2), with the latter one being able to accelerate the resolving phase of the disease.

Conclusion AnxA1 is an endogenous determinant for the therapeutic ef cacy of Dex in in ammatory arthritis. Such an effect can be partially mimicked by application of SuperAnxA1 which may represent the starting point for novel antiarthritic therapeutic strategies.

\section{INTRODUCTION}

There is genuine interest in detailing cellular and molecular events regulating the phase of the resolution of inflammation, with the characterisation of specific players and targets. ${ }^{1}$ This interest stems from the appreciation that, in ideal settings, a strong pro-inflammatory response, orchestrated by cytokines, adhesion molecules and chemoattractants ${ }^{2}$ must be followed by an anti-inflammatory and pro-resolving phase, which assures spatial and timely control of the response the host organises upon encounter with an insult, ${ }^{3}$ leading to resolution and regain of tissue functions.
Annexin A1 (AnxA1) is an effector of resolution. ${ }^{4}$ Highly expressed in immune cells (eg, polymorphonuclear cells and macrophages), this protein is externalised to exert paracrine and juxtacrine effects, the vast majority of which are mediated by the formylpeptide receptor type 2 (FPR2/ALX ([Lipoxin A4 receptor]) or FPR2, in rodents). ${ }^{5}$ Intriguingly, FPR2/ ALX is also the lipoxin $\mathrm{A}_{4}$ receptor ${ }^{6}$ indicating the existence of important - yet not fully appreciated - networks in resolution. ${ }^{7}$ Another receptor is also advocated to mediate the effects of AnxA1, the formyl-peptide receptor type 1 or FPR1 (FPR1 in rodents), 8 though it is unclear if and how this receptor binds the full-length protein ${ }^{9}$ or whether - more selectively - it would mediate effects of the AnxA1 peptidomimetic peptide Ac2-26. ${ }^{10}$

The majority of experimental approaches used to define properties of pro-resolving mediators have been reliant on models of acute inflammation, characterised by rapid polymorphonuclear influx followed by inflammatory monocytes which differentiate into tissue macrophages ${ }^{3} 11$ to phagocytose debris and apoptotic cells and favour tissue repair, a conclusive act in resolution that may require reepitheliation ${ }^{12}$ or a shift in fibroblast phenotype. ${ }^{13}$ In this context, the pro-resolving nature of AnxA1 has been demonstrated in a variety of experimental settings, noting induction of apoptosis, promotion of efferocytosis and tissue repair, spanning from models of acute inflammation $\left(\mathrm{eg},{ }^{14}{ }^{15}\right)$ to a model of colitis where delayed resolution was observed in AnxA1 ${ }^{-/-}$mice. ${ }^{16}$ An important aspect of AnxA1 biology is its second messenger role in the actions of glucocorticoids, a function mainly addressed in cellular settings $\left(\mathrm{eg},{ }^{17}\right)$ or in models of acute inflammation. ${ }^{18} 19$ However, Yang's group applied antigen-induced arthritis to observe reduction afforded by dexamethasone (Dex) on synovitis, soft tissue inflammation and cartilage erosion attenuated in AnxA1-/- mice. ${ }^{20}$

The $\mathrm{K} / \mathrm{BxN}$ serum-induced model of inflammatory arthritis is ideal for investigating the impact of specific pro-resolving pathways. Using this model of active phase of rheumatoid arthritis, Krönke et al reported a higher degree of joint inflammation in 12/15-lipoxygenase ${ }^{-/}$mice, associated with their inability to synthesise lipoxin $\mathrm{A}_{4} \cdot{ }^{21}$ Similarly, animals nullified for pro-resolving melanocortin receptor type 3 displayed higher degree of arthritis and delayed resolution, accompanied by augmented cytokine expression and osteoclast online under the BMJ Journals unlocked scheme, see http:// ard.bmj.com/info/unlocked.dtl 
responsiveness. ${ }^{22}$ An equally interesting study investigated the effect of absence of glucocorticoid signal in osteoblasts, by means of restricted transgenic expression of $11 \beta$-hydroxysteroid dehydrogenase type 2 , in this model of inflammatory arthritis. The outcome was that of a more attenuated arthritis in the transgenic mice when assessed macroscopically and histologically, and selectively in the delayed phase, after peak disease. ${ }^{23}$

The present study made use of the serum-induced model of inflammatory arthritis to: (1) monitor potential modulation of endogenous AnxA1, FPR1 and FPR2; (2) determine the effect of Dex in wild type and AnxA1 ${ }^{-/-}$mice; and (3) establish the pharmacological potential of exogenously administered AnxA1.

\section{MATERIALS AND METHODS}

\section{Animals}

BALB/c male 8-week old wild type AnxA1+/+ and AnxA1-/mice $^{24}$ were bred at Charles River (Kent, UK). All experiments were approved and performed under the guidelines of the Ethical Committee for the Use of Animals, Barts and The London School of Medicine and Home Office regulations (Scientific Procedures Act, 1986).

\section{Model of in ammatory arthritis} Arthritis induction

Mice received either 50+50 $\mu$ l (day 0 and 2) or $200 \mu \mathrm{l}$ (day 0) of arthritogenic $\mathrm{K} / \mathrm{BxN}$ serum. ${ }^{22}$ Disease was monitored by assessing the clinical score where a maximum of 12 points could be given per animal: 0 point, no evidence of inflammation; 1 point, subtle inflammation on metatarsal phalanges joints, individual phalanx; 2 points, swelling on dorsal or ventral surface of paw; 3 points, major swelling on all aspects of paw. ${ }^{25}$ Paw volume was assessed by water plethysmometry (Ugo Basile, Milan, Italy).

\section{Pharmacological intervention}

Mice were injected intraperitoneally with Dex (disodium salt; Sigma, Dorset, UK) prepared in sterile phosphate buffered saline (Sigma) and given at a dose range of 3-30 $\mu \mathrm{g}$ once daily after disease onset (from day 2). AnxA1 and SuperAnxA1 were generated as recently described ${ }^{26}$ and administered intraperitoneally, from day 2 , at $1 \mu \mathrm{g}$ per mouse. ${ }^{26}$

\section{Histology and immunohistochemistry Histology}

Joints were decalcified and paraffin embedded. Sections (4 micrometers) were stained with $\mathrm{H} \& \mathrm{E}$ or safranin $\mathrm{O}$ with fast green counterstain. Standard light microscopy was used to determine the degree of synovitis, pannus formation, cartilage and bone erosion which were graded from 0 (no disease) to 5 (severe) by two blinded examiners, as reported. ${ }^{22}$

\section{Immunohistochemistry}

An antigen retrieval step using citrate buffer ( $\mathrm{pH}$ 6.0) was performed and then sections were incubated with rabbit polyclonal anti-AnxA1 (1:1000) (Zymed Laboratories, Cambridge, UK) or anti-FPR2 (1:4000). ${ }^{27}$ After washing and the secondary biotinylated antibody (Dako, Cambridge, UK), staining was detected using a peroxidase conjugated streptavidin complex (Dako) and colour developed using 3,3' Diaminobenzidine (DAB) substrate (Sigma).

\section{Molecular analyses}

Ankle joints were snap frozen in liquid nitrogen and homogenised in Qiagen RLT plus buffer (Qiagen, Crawley, UK) using Precellys 24 ceramic bead homogenisation technology (Bertin
Technologies, Montigny-le-Bretonneux, France). RNA was extracted using RNeasy Plus mini kit (Qiagen) and genomic DNA contamination eliminated with Turbo DNA-free kit (Applied Biosystems, Foster City, California, USA). Complementary DNA was synthesised using SuperScriptIII reverse transcriptase and OligoDt primers (Invitrogen). Quantitative real-time PCR was performed using QuantiTect primers (Qiagen) and ABI Prism 7900 sequence detector system (Applied Biosystems). RQ values were calculated using the $\left.2^{-(} \Delta \Delta^{\mathrm{C} t}\right)$ method and normalised to an individual naive AnxA1 ${ }^{+/+}$or $A n x A 1^{-/-}$mouse (calibrator sample).

All $\mathrm{Ct}$ values were normalised to endogenous Gapdh gene product. Since factors such as hypoxia can influence Gapdh gene levels, ${ }^{28}$ a second house-keeping gene Rpl32 was used to normalise and confirm data (see table S2).

\section{Statistics}

Data are expressed as mean \pm SE. Studentttestwas used to compare two groups. Comparison of clinical scores and paw volumes 3 were made using 2-way analysis of variance. A value of $p<0.05$ was considered significant.

\section{RESULTS}

\section{Characterisation of joint in ammation}

We began this study to optimise the model by comparing two different protocols: $200 \mu 1$ of serum at day $0\left(\operatorname{as~in~}^{22}\right)$ or $50+50$ $\mu \mathrm{l}$ at day 0 and day 2. A remarkably similar arthritic response could be measured with respect to disease profile and intensity (figure 1A). High levels of disease were achieved as early as day 2 postinjection with maximal response by day 6 , after which the disease resolved to almost normal by day 20 . Incidence of disease was similar between the two volumes of $\mathrm{K} / \mathrm{BxN}$ serum (table S1). The arthritic response was mirrored by changes in paw volume with similar profiles with no difference between the two protocols (figure 1B).

Microscopic analyses of non-arthritic ankle joints showed an acellular articular cavity with intact unicellular synovial lining and uniform cartilage structure (figure $1 \mathrm{C}$, top panel). In contrast, arthritic joints from both $50+50 \mu \mathrm{l}$ and $200 \mu \mathrm{K} / \mathrm{BxN}$ arthritis groups displayed marked cell infiltration within a thickened synovial lining. Pannus invasion leading to cartilage erosion was observed and proteoglycan loss was confirmed with safraninO (figure 1C, middle and bottom panels). Quantitative histological scoring showed no significant difference between the two groups of arthritic mice (figure 1D).

The ankle is the predominant joint to be affected in this model of inflammatory arthritis, yet we monitored structural changes in the knee, as is perhaps more relevant to human disease; we observed that knee joints displayed mild synovitis accompanied with thickening of synovial lining, pannus formation and marked cartilage erosion (see figure S1). Based on these results, in agreement with a recent published study, ${ }^{29}$ the $50+50 \mu \mathrm{l}$ serum protocol was selected for subsequent experimentation.

\section{Expression of AnxA1 and FPR2 in the joint}

At the mRNA level, a low degree of expression for both FPR1 and FPR2 could be measured, with high AnxA1 message content (see table S2). These results were partially confirmed by protein analyses, since AnxA1 was mildly expressed and localised predominantly in the synovial lining, while FPR2 was localised to the periosteal lining with very little detection in synovium (figure 2A). In arthritic joints, AnxA1 and FPR2 expression was 


\section{Figure 1}
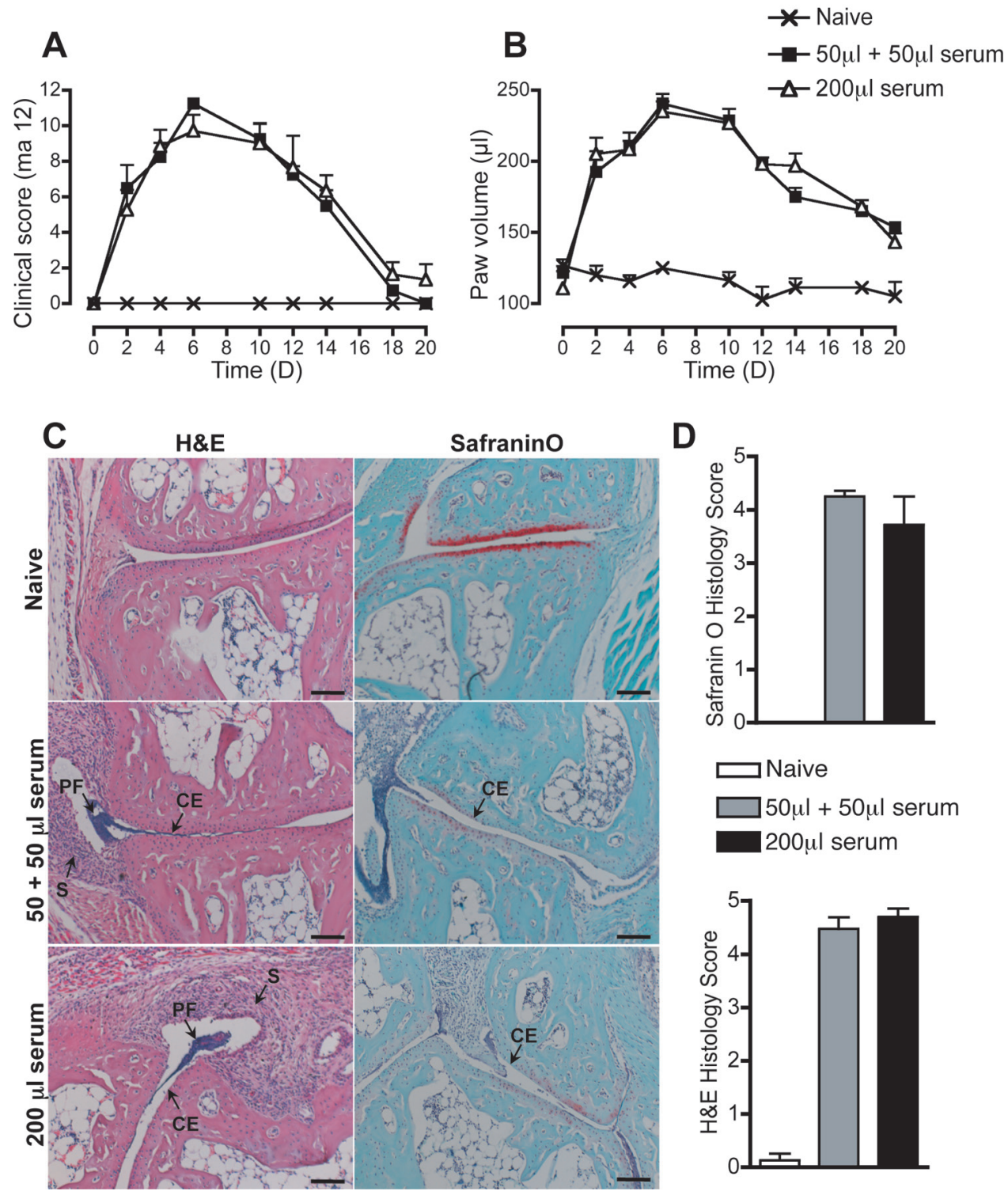

Figure 1 Characterisation of the arthritic response to low volume $K / B x N$ serum. Mice $(n=8)$ were given an intraperitoneal injection of $50 \mu l$ at day 0 and day $2(50+50 \mu \mathrm{l})$ or a single injection of $200 \mu \mathrm{l}$ at day 0 of K/BxN serum. (A) Arthritic scores and (B) paw oedema were monitored over the time course. No signi cant difference (two-way analysis of variance) was observed between the two K/BxN serum groups. (C) Ankles of naive, $50+50$ $\mu \mathrm{l}$ and $200 \mu \mathrm{K} / \mathrm{BxN}$ serum group. Decalci ed paraf $\mathrm{n}$ embedded joints were stained by H\&E and safraninO. Representative images are shown; scale bars, $50 \mu \mathrm{m}$. CE, cartilage erosion; PF, pannus formation; S, synovitis. (D) Histomorphometric analyses of joint sections. All results are expressed as mean $\pm S E$.

highly detectable within the synovial lining (FPR2) and tissue (AnxA1 and FPR2) (figure 2B). Antibody controls and AnxA1 $1^{--}$ joints did not show any staining (figure 2C,D).

\section{Dex is inactive in AnxA1-/- mice}

Despite wide application of this model of inflammatory arthritis, the bioactions of glucocorticoids have been scantly investigated. We selected the 10- $\mu$ g dose of Dex from initial studies (figure S2). Administration of Dex to AnxA1 $1^{+/+}$mice exerted profound inhibitory effects with $\sim 60 \%$ inhibition of the severity of arthritis at day 6 and $~ 80 \%$ at day 10 compared with vehicle-treated mice (figure $3 \mathrm{~A}$ ). AnxA1 ${ }^{-/-}$mice displayed a similar profile of arthritis to AnxA1 $1^{+/}$animals. However, the antiarthritic effect of Dex was absent in animals nullified for AnxA1 (figure 3A). Analysis of presence of severe disease (score $\geq 8$ ) indicated a trend for higher incidence in AnxA1-/mice and a marked reliance on endogenous AnxA1 for the protective effects of Dex (table S3).

Histological analyses were in line with arthritic scores. AnxA1 $1^{+/+}$mice displayed marked synovitis accompanied by pannus formation and cartilage destruction (figure $3 \mathrm{~B}$, left panels). Treatment with Dex prevented signs of disease (figure 3B, middle panels), while AnxA1 $1^{-/}$mice treated with vehicle control (not shown) and Dex (figure $3 \mathrm{~B}$, right panels) showed similar disease pathology to AnxA1 ${ }^{+/+}$mice. These structural changes were reflected in the histological scores with $~ 75 \%$ inhibition afforded by Dex only in AnxA1 ${ }^{+/+}$mice (figure 3C). 


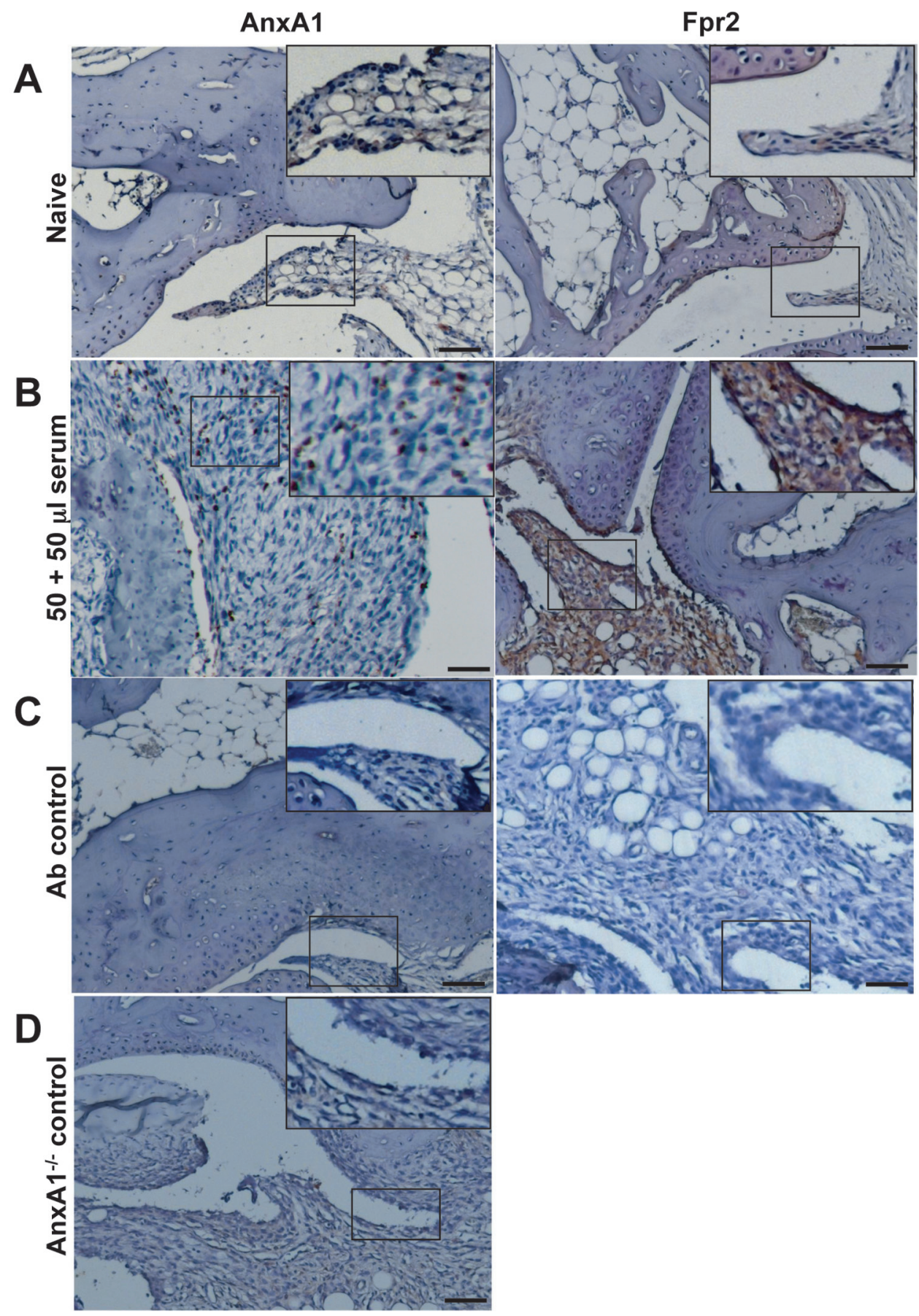

Figure 2 Protein expression of AnxA1 and FPR2 in ankle joints of naive mice. Ankles of naive and K/BxN serum (50+50 $\mu \mathrm{l}$, day 0 and day 2$)$ were processed (see methods) and stained for AnxA1 and FPR2, with a haematoxylin counterstain. Images representative of three distinct analyses in four mice per status are shown. (A) Naive mouse ankle joints. (B) Serum-treated mouse ankle joint. (C) Same joints but tested without the primary antibody. (D) Joint of an AnxA1 ${ }^{-/-}$mouse acting as negative control for AnxA1 staining. Scale bars, $20 \mu \mathrm{m}$.

Analyses of inflammatory gene messages, normalised to Gapdh and Rpl32 (see figure S3) and selected from our previous study, ${ }^{22}$ revealed that in wild type mice Dex inhibited Cxcl10, Nos2, interleukin (IL)-1 $\beta$ and Ccl2. Tumour necrosis factor $\alpha$ (TNF $\alpha$ ) was inhibited by $\sim 25 \%$ while expression of the chemokine receptor $\mathrm{Cxcr3}$ was not modulated (figure $3 \mathrm{D}$ ). These inhibitory effects of Dex were absent or significantly reduced in AnxA1 $1^{-1-}$ mice.
Next, we monitored expression of AnxA1 and its receptors, normalised to Gapdh and Rpl32 (see figure S4). In arthritic AnxA1+/+ mice, both FPR1 and FPR2 mRNA expression was enhanced over naive joints (figure 4), a finding which is in line with FPR2 protein expression (see figure 2). Dex decreased both FPR1 and FPR2 message with little comparative effect on AnxA1 (figure 4). In mice devoid of AnxA1, a >10-fold increase in FPR1 expression was observed comparing arthritic to naive joints, with FPR2 remaining 
A

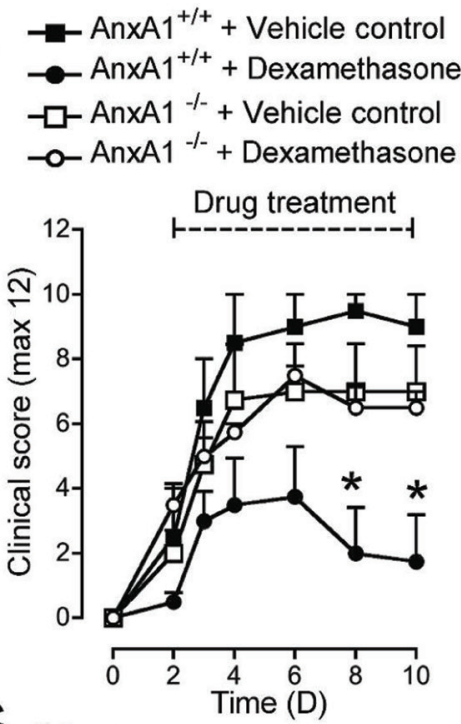

C

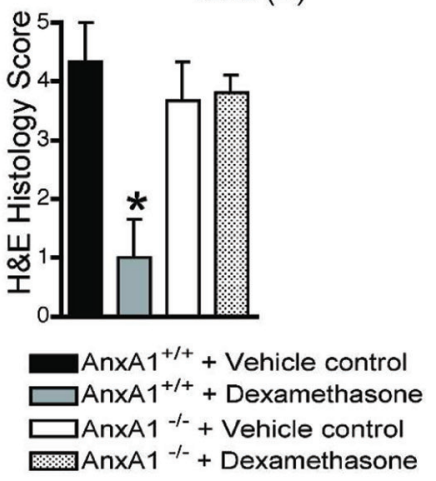

B
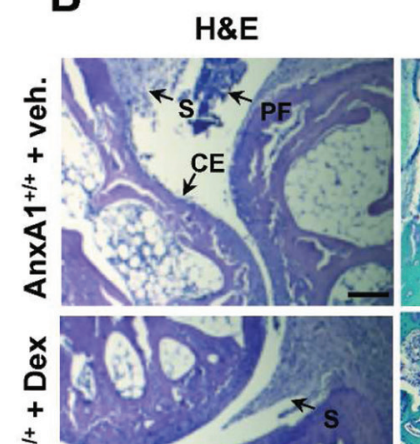

声

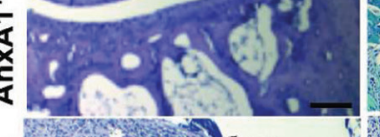

S.
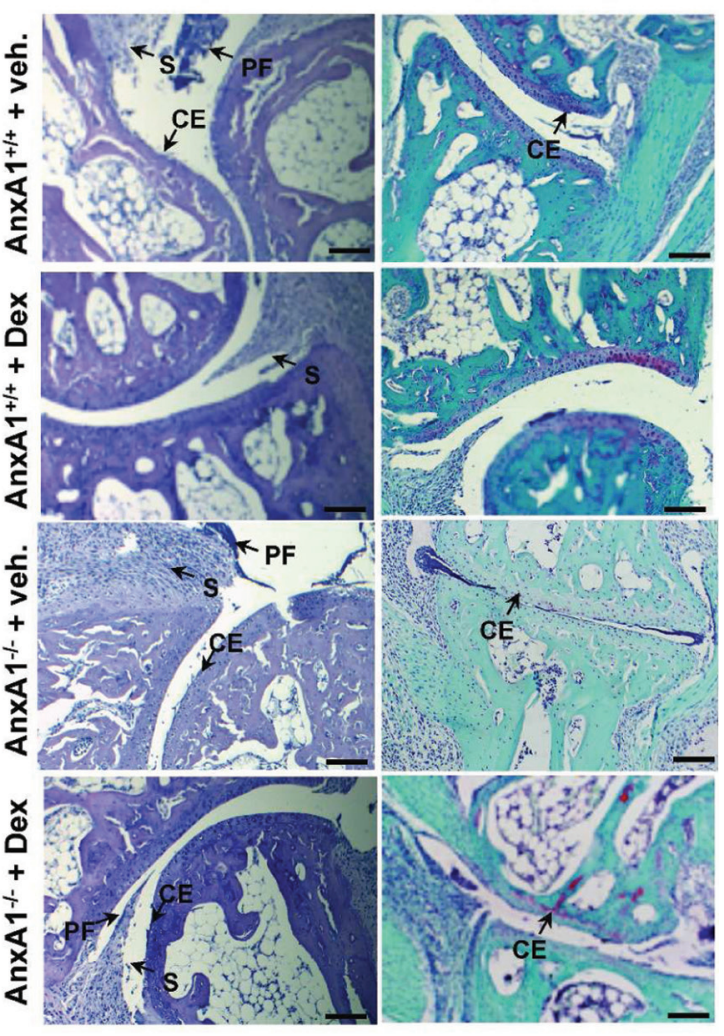

D

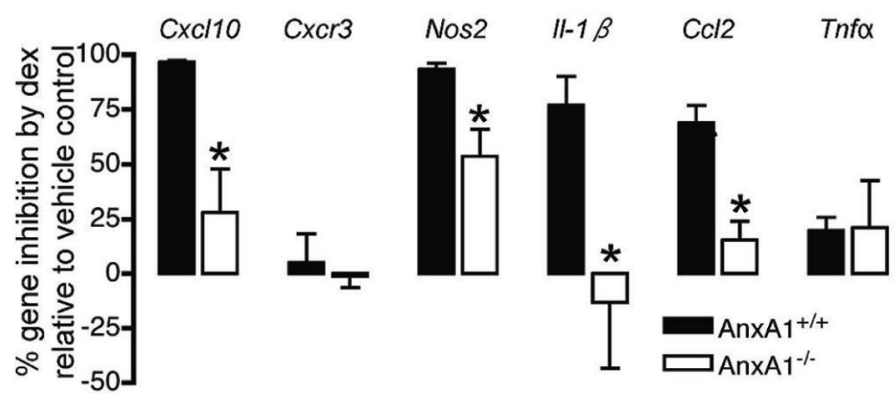

Figure 3 Dexamethasone (Dex) attenuates $\mathrm{K} / \mathrm{BxN}$ arthritis in $\mathrm{AnxA} 1^{+/+}$but not $\mathrm{AnxA} 1^{-/-}$mice. AnxA1 ${ }^{+/+}$and $\mathrm{AnxA} 1^{-/-}$mice were given an intraperitoneal injection of $\mathrm{K} / \mathrm{BxN}$ serum $(50 \mu \mathrm{l}$ at day 0 and day 2$)$ and then received vehicle or Dex (10 $\mu \mathrm{g}$ intraperitoneally daily). (A) Arthritic scores as monitored over a 10-day time course. ${ }^{*} p<0.05$ versus appropriate vehicle control (two-way analysis of variance). (B) Right ankles of mice from each experimental group were taken at day 10 and joints processed for staining by H\&E and safraninO. Representative images are shown. Scale bars, $50 \mu \mathrm{m}$. CE, cartilage erosion; PF, pannus formation; S, synovitis. (C) Histomorphometric analyses of joint sections. ${ }^{*} p<0.05$ versus appropriate vehicle control (Student t test). (D) Quantitative real-time PCR was performed on cDNA from left ankle joints; pro-in ammatory genes were analysed with all $\mathrm{Ct}$ values normalised to endogenous Gapdh (four mice per group). R0 values were calculated using $2-(\Delta \Delta \mathrm{Ct})$ and data shown here as mean $\%$ gene inhibition by Dex relative to vehicle control groups \pm SE. Naive joints were set as the calibrator samples. ${ }^{*} p<0.05$ versus respective vehicle control (Student t test).

at a similar expression. In absence of AnxA1, Dex could not regulate either FPR1 or FPR2 gene expression (figure 4).

\section{SuperAnxA1 accelerates resolution of the arthritic joint}

In the final set of experiments, we assessed the pharmacological potential of these findings. Proteinase 3 (PR3) may limit AnxA1 functions in neutrophilic inflammation, cleaving it at the $\mathrm{N}$-terminal region. Thus, we monitored the profile of PR3 gene in the arthritic joint and found it significantly rose from baseline (day 0) to peak of disease (day 6) (figure 5 and figure S5). This observation, coupled with abundant expression of FPR1 and FPR2 in the joints justified the testing of SuperAnxA1. Dosed at $1 \mu \mathrm{g}$ per mouse, AnxA1 was not particularly effective in reducing disease (figure $5 \mathrm{~B}$ ). On the other hand, cleavage-resistant SuperAnxA1 did not affect disease progress (eg, from day 2 to day 6) but significantly accelerated resolution (figure $5 \mathrm{~B}$ ), with $50 \%$ less disease incidence compared with controls (table 1).

The macroscopic joint protection afforded by SuperAnxA1 was confirmed microscopically, with preservation of cartilage structure with little loss of proteoglycans (figure 5C, middle 


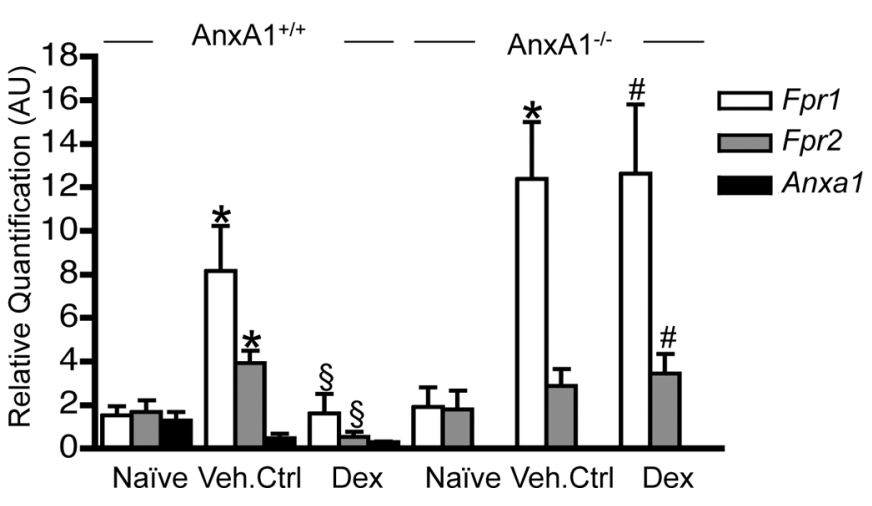

Figure 4 Pro le of AnxA1, FPR1 and FPR2 gene product expression in ankle joints of $A n \times A 1^{+/+}$and $A n \times A 1^{-/-}$mice treated with dexamethasone (Dex). AnxA1 ${ }^{+/+}$and $A n \times A 1^{-/-}$mice were given an intraperitoneal injection of $\mathrm{K} / \mathrm{BxN}$ serum $(50 \mu \mathrm{l}$ at day 0 and day 2$)$ and then received vehicle or Dex (10 $\mu \mathrm{g}$ intraperitoneally daily). Quantitative real-time PCR was performed on cDNA from left ankle joints of mice from each experimental group (day 10). The AnxA1, FPR1 and FPR2 genes were analysed with all Ct values normalised to endogenous Gapdh (four mice per group). Naive joints were set as the calibrator samples. Mean $\mathrm{RO}$ values \pm SE shown here were calculated using $2-(\Delta \Delta \mathrm{Ct})$ method. ${ }^{*} p<0.05$ versus naive; $p<0.05$ versus appropriate vehicle control; $\# p<0.05$ versus $A n \times A 1^{+/+}$group (Student $t$ test).

panels). Synovitis was apparent within these joints, but substantially lower than controls (figure 5C, top panels). The joints of AnxA1 treated mice were disrupted to a similar extent as controls (figure 5C, bottom panels). Quantitative analysis of damage within the ankle joints confirmed the observational studies (figure 5D). Finally, ankle joints from vehicle- and SuperAnxA1treated mice demonstrated reduced expression of the Cxcl10 and Cxcr3 pair, as well as Nos2, IL-1 $\beta$, Ccl2, IL- 6 but not IL-10 and TNF $\alpha$ (figure S6).

\section{DISCUSSION}

AnxA1 is the prototype of a new class of mediators able to potently modulate both the innate and adaptive immune response; while effecting inhibitory properties on inflammatory cells, setting in motion pro-resolving responses, this protein acts as a positive relay in $\mathrm{T}$ cell activation. ${ }^{4}$ As an example, exogenous and endogenous AnxA1 potentiate T cell receptor-downstream signalling ${ }^{30}$ and assure proper dendritic cell-mediated $\mathrm{T}$ cell clonal expansion. ${ }^{31}$ In agreement with these dual functions, AnxA1 ${ }^{-/}$mice experience augmented acute inflammatory responses, ${ }^{1415}$ yet display reduced degree of tissue damage in an experimental model of multiple sclerosis. ${ }^{32}$ Less clear is the role of the protein in models of arthritis.

In the collagen-induced arthritis model, AnxA1 injection during the immune phase (within 1 week postcollagen immunisation) led to higher disease as emerged 3 weeks later. ${ }^{30}$ In a model of antigen-induced arthritis, assessed at a single time-point (day 7), endogenous AnxA1 modulated some of the anti-inflammatory effects of Dex, including inhibition of pro-inflammatory genes including IL-1 $\beta$ and TNF $\alpha .{ }^{20}$ No studies have yet investigated the role of endogenous or exogenous AnxA1 in the seruminduced inflammatory arthritis model.

$\mathrm{K} / \mathrm{BxN}$ serum-induced arthritis ${ }^{33} 34$ is a versatile model that can be applied to transgenic mice with little dependence on strain. ${ }^{35}$ This model addresses the contribution of neutrophils and macrophages to inflammatory arthritis, as evident from depletion studies. $^{36} 37$ Conversely, this experimental arthritis does not rely on $\mathrm{T}$ and $\mathrm{B}$ cells as demonstrated with the $\mathrm{RAG}^{-/-}$mice ${ }^{28}$ which, in contrast, display delayed disease onset and severity in collagen-induced arthritis. ${ }^{38}$ Here we describe that low dosage arthritogenic serum provokes a high degree of arthritis and disease incidence, data which are in agreement with a recent study by Boilard's group. ${ }^{29}$ We noted a remarkable joint disruption and report a novel parallelism between the ankle and knee joint. Marked synovitis associated with disruption of cartilage occurs along a well-defined time-profile with peak disease at day 6 followed by a resolution phase. ${ }^{22} 35$ Use of transgenic mice has allowed some definition of the disease pathogenesis, revealing a fundamental role for myeloid cells ${ }^{36} 37$ and IL-1..$^{39} 40$ More recently, this model of arthritis has been applied to proresolving pathways, noting that 12/15-lipoxygenase or melanocortin type-3 receptor deficient mice experience a higher degree of disease. ${ }^{21} 22$

AnxA1 is highly abundant in myeloid and stromal cells and its expression can be modulated by glucocorticoids and other antiinflammatory drugs. ${ }^{41}$ In addition, cell activation such as adhesion or extravasation also engages the AnxA1 system as a way to activate an inhibitory circuit to modulate the inflammatory response. ${ }^{27}$ After several pharmacological investigations with AnxA1 or mimetics, AnxA1 null mice were produced and tested in models of acute inflammation ${ }^{1524}$ revealing important modulatory functions. ${ }^{4}$ As discussed above, AnxA1 role in chronic inflammatory settings in less defined.

This study began by detecting protein and mRNA of elements of the AnxA1 pathway during ongoing inflammatory arthritis, reporting a high degree of expression for AnxA1, FPR1 and FPR2 both in resting and arthritic joints. At the protein level, AnxA1 localisation in the mouse synovium seems to parallel that observed in the human rheumatoid arthritis synovium. ${ }^{42}$ However, at least in the conditions applied here, lack of AnxA1 does not seem to impact on the profile of disease. Yet, it remains to be seen whether such a lack of function would also be seen in knockouts for the AnxA1 receptors. ${ }^{54}$ As an example, in preliminary observations, mice nullified for FPR2 displayed higher arthritic score upon injection of the arthritogenic serum. ${ }^{44}$

Glucocorticoids have been scarcely tested in this model. Buttgereit et al have tested the effect of osteoblast-restricted absence of glucocorticoid signalling on disease outcome, noting a worsening with marked joint disruption. ${ }^{23}$ In these mice, bone cells could not respond to glucocorticoids from birth; thus, it is possible that developmental aspects might be relevant and subtle long-term alterations occur. For instance, it is known that glucocorticoids exert a permissive effect on important cytokine function in the liver, by assuring proper expression of the IL- 6 and IL-1 receptors. ${ }^{45} 46$ IL- 6 is also a master-cytokine for osteoblasts (and fibroblasts) and so a malfunctioning or inadequate glucocorticoid signalling, prolonged from birth, may generate responses that are not necessarily replicated by acute glucocorticoid treatment.

In keeping with this, our pharmacological analyses with doses of Dex as low as $10 \mu \mathrm{g}$ exerted potent protective effects on the joint, evident both as inhibition of pannus formation and chondro-protection. It was striking to observe that the effects of the glucocorticoid were lost in AnxA1 ${ }^{-/}$animals. Thus, while AnxA1 ${ }^{-/-}$mice did not experience a difference in the extent and profile of arthritis, at variance from the antigen-induced arthritis model, ${ }^{20}$ endogenous AnxA1 still proved important in the inhibitory effects of Dex on a discrete set of pro-inflammatory genes, of which IL-1 $\beta$, Cxcl10 and $\mathrm{Ccl} 2$ were of particular interest. Selective modulation of gene expression downstream AnxA1 is in keeping with the observations made in the antigen-induced 
A
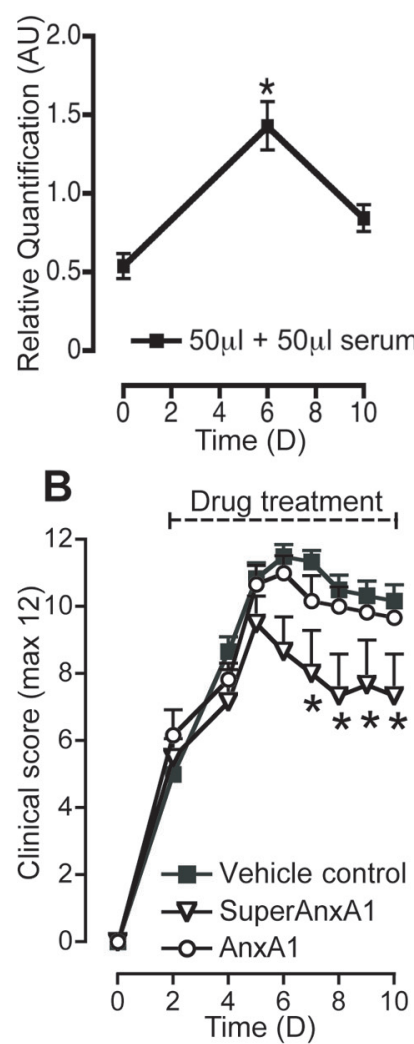

C
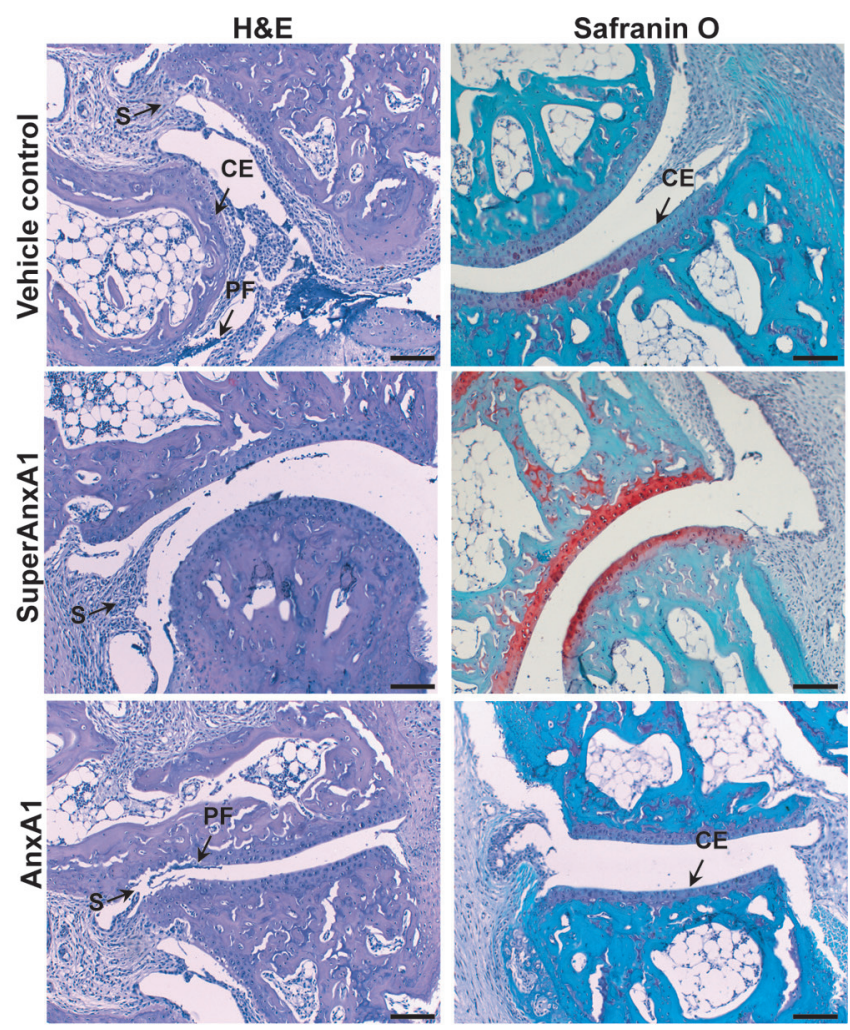

D

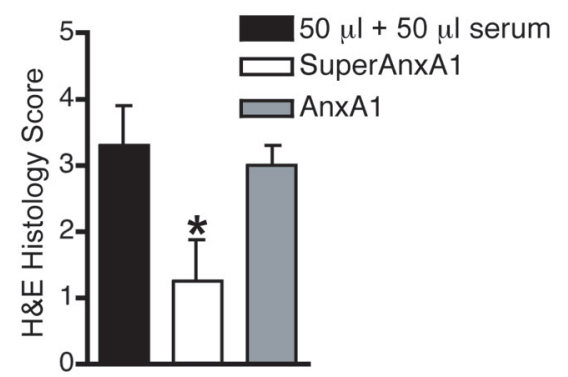

Figure 5 SuperAnxA1 accelerates resolution of $K / B x N$ arthritis. AnxA $1^{+/+}$mice $(n=6)$ were given an intraperitoneal injection of $K / B x N$ serum $(50$ $\mu l$ at day 0 and day 2 ) and then received vehicle, human recombinant AnxA1 or SuperAnxA1 (1 $\mu$ g intraperitoneally daily from day 2). (A) PR3 gene product expression was analysed with $\mathrm{Ct}$ values normalised to endogenous Gapdh. $\mathrm{R} 0$ values were calculated using $2-(\Delta \Delta \mathrm{Ct})$ and data shown here as mean \pm SE. Naive mice (D0) were set as the calibrator samples. ${ }^{*} p<0.05$ versus naive (Student $t$ test). (B) Arthritic score. ${ }^{*} p<0.05$ versus vehicle control (two-way analysis of variance). (C) Right ankles of mice from each experimental group were taken at day 10 and joints processed for staining by H\&E and safraninO. Representative images are shown. Scale bars, $50 \mu \mathrm{m}$. CE, cartilage erosion; PF, pannus formation; $\mathrm{S}$, synovitis. (D) Histomorphometric analyses of joint sections. ${ }^{*} p<0.05$ versus vehicle control (Student $t$ test).

Table 1 SuperAnxA1 reduces the severity of in ammatory arthritis

\begin{tabular}{lccr}
\hline Time (days) & Vehicle control & SuperAnxA1 & \multicolumn{1}{c}{ AnxA1 } \\
\hline 0 & $0 \%(0 / 6)$ & $0 \%(0 / 6)$ & $0 \%(0 / 6)$ \\
6 & $100 \%(6 / 6)$ & $83.3 \%(5 / 6)$ & $100 \%(6 / 6)$ \\
10 & $100 \%(6 / 6)$ & $50 \%(3 / 6)$ & $100 \%(6 / 6)$ \\
\hline
\end{tabular}

Data report the number of mice with a severe arthritic score taking a threshold of $\geq 8$. SuperAnxA1 or AnxA1 was given from day 2 at daily doses of $1 \mu \mathrm{g}$ intraperitoneally; the $\mathrm{K} / \mathrm{BxN}$ arthritogenic serum was given on day 0 and day 2 at volumes of $50 \mu \mathrm{l}$ each time.

arthritis model. ${ }^{20}$ The fundamental role that IL-1 $\beta$ exerts in this model of inflammatory arthritis has been addressed through a variety of genetic approaches targeting directly the cytokine, its receptor ${ }^{39} 47$ or endogenous antagonist. ${ }^{40}$
Modulation of gene expression downstream of endogenous AnxA1 can also be discussed in relation to the modulation of genes of the AnxA1 pathway. In fact, Dex did not appear to induce AnxA1 or FPR gene expression at the level of the joint, yet was highly reliant on endogenous AnxA1 both with respect to cell recruitment and inflammatory gene expression. We reason that these data indicate multiple functions of endogenous AnxA1, which become of relevance only after administration of the glucocorticoid.

The lack of correlation between AnxA1 gene product and protein is intriguing though it should be said that glucocorticoidregulation of the AnxA1 pathway is multifaceted; thus, nongenomic modulation ${ }^{48}$ with release of the protein, for instance, could account for apparent lower tissue levels of the AnxA1 protein. AnxA1 analysis, at a single time point, could also be confounded by genomic regulation exerted by glucocorticoids 1549 
and other factors present in an inflamed joint such as IL- $6^{50}$ and TNF. ${ }^{51}$ Clearly, the current lack of characterisation of the AnxA1 promoter contributes to this poor understanding of the mechanisms operative in these settings. Moreover, the fact that glucocorticoids like Dex induce AnxA1 gene in innate immune cells $^{14} 15$ while downregulating it in adaptive immune cells ${ }^{52}$ adds another layer of complexity to this issue which inevitably requires further experimentation.

The versatility of this model of inflammatory arthritis justifies its application to a variety of targets; in line with the major infiltration of neutrophils and mast cells, ${ }^{53}$ we could observe dynamic expression of PR3 gene product in the arthritic joint. PR3 is emerging as an important effector of neutrophilic inflammation $^{54}$ and can cleave, among a variety of substrates, also AnxA1. ${ }^{55}$ Therefore, we concluded this study by testing the potential of AnxA1 mimetics, comparing the effect of native protein with that of SuperAnxA1, a mutant resistant to serine protease-induced cleavage. ${ }^{26}$ Administered at the dose of $1 \mu \mathrm{gg}$ (corresponding to $27 \mathrm{pmol}$ ), SuperAnxA1, but not AnxA1, attenuated joint disease. It was interesting to note that SuperAnxA1 did not impact on the induction of arthritis yet it accelerated the resolution process so that by day 10 a preserved joint structure could be observed. We wish to propose that higher resistance to proteolysis might underlie its pharmacological efficacy, though we cannot exclude the confounding element of potential differences in pharmacokinetics. In any case, these results provide strong proof-of-concept that an AnxA1 derivative, resistant to proteolysis, could be beneficial in modulating joint inflammation. SuperAnxA1 could be the backbone for future strategies aiming at capitalising AnxA1 biology for novel antiarthritic approaches.

Glucocorticoids are of wide clinical use because of their ability to impact several effectors of arthritis, with a recent appreciation that, besides inhibition of pro-inflammatory gene expression, gene induction is also of therapeutic relevance. ${ }^{56}$ 57 In the context of chronic inflammatory diseases, induction of dual-specificity phosphatase $1^{58}$ and glucocorticoid-induced leucine zipper $^{59}$ genes could be of mechanistic value. In addition, we show here that endogenous AnxA1 is also relevant to the antiarthritic effect of low-dose Dex, yet we cannot demonstrate induction of this gene at the level of the arthritic joint leading to our proposal that the protein is either modulated in a non-genomic fashion by this glucocorticoid ${ }^{4160}$ or the regulation occurs outside the site of the arthritic joint, an obvious site being the vasculature and the control of the egress of bloodborne neutrophils. ${ }^{4}$ Analysis of AnxA1 expression in distant tissues (eg, blood) during ongoing arthritis and glucocorticoid treatment can shed light on this point, which has also been touched upon above in relation to lack of correlation between joint AnxA1 mRNA and protein expression.

Collectively, this study reveals potent antiarthritic properties for Dex in a model of inflammatory arthritis, using very low doses perhaps reminiscent of clinical application on low-dose glucocorticoids in $\mathrm{RA}^{61}$ and, in terms of drug discovery programmes, justifies the testing of AnxA1 mimetics to control the active phase of the disease.

Contributors HBP, performed experiments, analysed data, wrote manuscript. KNK, performed experiments, analysed data. ALFS, performed experiments, analysed data. FDA, provided unique tools, planned experiments. MPS, analysed data. APG, performed experiments, analysed data. MG, planned project, provided unique tool. $\mathrm{CP}$, planned project, managed project. SMO, performed experiments, analysed data, wrote draft of the manuscript. MP, planned and managed project, wrote manuscript.

Funding This work was supported by a Wellcome Trust (UK) project grant 083551. SMO is funded by Funda o de Amparo Pesquisa do Estado de S o Paulo FAPESP
(Grant 2011/00128-1) and Conselho Nacional de Desenvolvimento Cient' co e Tecnol gico CNPq (Grant 302768/2010-6)

Competing interests None.

Provenance and peer review Not commissioned; externally peer reviewed.

\section{REFERENCES}

1. Gilroy DW, Lawrence T, Perretti M, et al. In ammatory resolution: new opportunities for drug discovery. Nat Rev Drug Discov 2004;3:401 16

2. Ley $\mathbf{K}$, Laudanna C, Cybulsky Ml, et al. Getting to the site of in ammation: the leukocyte adhesion cascade updated. Nat Rev Immunol 2007; 7:678 89.

3. Serhan CN, Brain SD, Buckley CD, et al. Resolution of in ammation: state of the art, de nitions and terms. FASEB J 2007;21:325 32

4. Perretti M, D Acquisto F. Annexin A1 and glucocorticoids as effectors of the resolution of in ammation. Nat Rev Immunol 2009;9:62 70.

5. Ye RD, Boulay F, Wang JM, et al. International Union of Basic and Clinical Pharmacology. LXXIII. Nomenclature for the formyl peptide receptor (FPR) family. Pharmacol Rev 2009;61:119 61

6. Chiang $\mathbf{N}$, Serhan CN, Dahl n SE, et al. The lipoxin receptor ALX: potent ligand-speci c and stereoselective actions in vivo. Pharmacol Rev 2006;58:463 87

7. Perretti M, Chiang N, La M, et al. Endogenous lipid- and peptide-derived anti-in ammatory pathways generated with glucocorticoid and aspirin treatment activate the lipoxin A4 receptor. Nat Med 2002;8:1296 302.

8. Walther A, Riehemann K, Gerke V. A novel ligand of the formyl peptide receptor: annexin I regulates neutrophil extravasation by interacting with the FPR. Mol Cell 2000;5:831 40

9. Hayhoe RP, Kamal AM, Solito E, et al. Annexin 1 and its bioactive peptide inhibit neutrophil-endothelium interactions under ow: indication of distinct receptor involvement. Blood 2006;107:2123 30

10. Ernst S, Lange C, Wilbers A, et al. An annexin $1 \mathrm{~N}$-terminal peptide activates leukocytes by triggering different members of the formyl peptide receptor family. $\mathrm{J}$ Immunol 2004;172:7669 76.

11. Serhan CN. Novel lipid mediators and resolution mechanisms in acute in ammation: to resolve or not? Am J Pathol 2010;177:1576 91.

12. Babbin BA, Lee WY, Parkos CA, et al. Annexin I regulates SKCO-15 cell invasion by signaling through formyl peptide receptors. J Biol Chem 2006;281:19588 99.

13. Muf ey LA, Zhu KO, Engrav LH, et al. Spatial and temporal localization of the melanocortin 1 receptor and its ligand $\alpha$-melanocyte-stimulating hormone during cutaneous wound repair. J Histochem Cytochem 2011;59:278 88.

14. Damazo AS, Yona S, D Acquisto F, et al. Critical protective role for annexin 1 gene expression in the endotoxemic murine microcirculation. Am J Pathol 2005; 166:1607 17

15. Damazo AS, Yona S, Flower RJ, et al. Spatial and temporal pro les for anti-in ammatory gene expression in leukocytes during a resolving model of peritonitis. J Immunol 2006;176:4410 18

16. Babbin BA, Laukoetter MG, Nava $P$, et al. Annexin $A 1$ regulates intestinal mucosal injury, in ammation, and repair. J Immunol 2008;181:5035 44

17. 0 Sullivan TP, Vallin KS, Shah ST, et al. Aromatic lipoxin A4 and lipoxin B4 analogues display potent biological activities. J Med Chem 2007;50:5894 902.

18. Getting SJ, Flower RJ, Perretti M. Inhibition of neutrophil and monocyte recruitment by endogenous and exogenous lipocortin 1. Br J Pharmacol 1997;120: 107582.

19. Yang $\mathbf{Y}$, Leech $\mathbf{M}$, Hutchinson $P$, et al. Antiin ammatory effect of lipocortin 1 in experimental arthritis. Inflammation 1997;21:583 96.

20. Yang YH, Morand EF, Getting SJ, et al. Modulation of in ammation and response to dexamethasone by Annexin 1 in antigen-induced arthritis. Arthritis Rheum 2004:50:976 84

21. Kr nke G, Katzenbeisser J, Uderhardt S, et al. 12/15-lipoxygenase counteracts in ammation and tissue damage in arthritis. J Immunol 2009;183 33839.

22. Patel HB, Bombardieri M, Sampaio AL, et al. Anti-in ammatory and antiosteoclastogenesis properties of endogenous melanocortin receptor type 3 in experimental arthritis. FASEB J 2010;24:4835 43

23. Buttgereit $\mathbf{F}$, Zhou $H$, Kalak $\mathrm{R}$, et al. Transgenic disruption of glucocorticoid signaling in mature osteoblasts and osteocytes attenuates $\mathrm{K} / \mathrm{BxN}$ mouse serum-induced arthritis in vivo. Arthritis Rheum 2009;60:1998 2007.

24. Hannon R, Croxtall JD, Getting SJ, et al. Aberrant in ammation and resistance to glucocorticoids in annexin 1-/- mouse. FASEB J 2003;17:253 5 .

25. Lee DM, Friend DS, Gurish MF, et al. Mast cells: a cellular link between autoantibodies and in ammatory arthritis. Science 2002;297:1689 92.

26. Pederzoli-Ribeil M, Maione F, Cooper D, et al. Design and characterization of a cleavage-resistant Annexin A1 mutant to control in ammation in the microvasculature. Blood 2010;116:4288 96.

27. Gastardelo TS, Damazo AS, Dalli J, et al. Functional and ultrastructural analysis of annexin $\mathrm{A} 1$ and its receptor in extravasating neutrophils during acute in ammation. Am J Pathol 2009;174:177 83. 
28. Zhong H Simons JW. Direct comparison of GAPDH, beta-actin, cyclophilin, and 28S rRNA as internal standards for quantifying RNA levels under hypoxia. Biochem Biophys Res Commun 1999;259:523 6.

29. Boilard E, Lai Y, Larabee K, et al. A novel anti-in ammatory role for secretory phospholipase A2 in immune complex-mediated arthritis. EMBO Mol Med 2010;2:172 87

30. D Acquisto F, Merghani A, Lecona E, et al. Annexin-1 modulates T-cell activation and differentiation. Blood 2007;109:1095 102.

31. Huggins A, Paschalidis N, Flower RJ, et al. Annexin-1-de cient dendritic cells acquire a mature phenotype during differentiation. FASEB J 2009;23:985 96

32. Paschalidis $\mathbf{N}$, Huggins $A$, Rowbotham NJ, et al. Role of endogenous annexin-A1 in the regulation of thymocyte positive and negative selection. Cell Cycle 2010;9:784 93.

33. Kouskoff V, Korganow AS, Duchatelle V, et al. Organ-speci c disease provoked by systemic autoimmunity. Cell 1996;87:811 22.

34. Matsumoto I, Staub A, Benoist C, et al. Arthritis provoked by linked T and B cell recognition of a glycolytic enzyme. Science 1999;286:1732 5.

35. Ji H, Gauguier D, Ohmura K, et al. Genetic in uences on the end-stage effector phase of arthritis. J Exp Med 2001;194:321 30.

36. Wipke BT, Allen PM. Essential role of neutrophils in the initiation and progression of a murine model of rheumatoid arthritis. J Immunol 2001:167:1601 8.

37. Solomon S, Rajasekaran N, Jeisy-Walder E, et al. A crucial role for macrophages in the pathology of K/B x N serum-induced arthritis. Eur J Immunol 2005:35:3064 73 .

38. Plows D, Kontogeorgos G, Kollias G. Mice lacking mature T and B lymphocytes develop arthritic lesions after immunization with type II collagen. J Immunol 1999;162:1018 23

39. Ji H, Pettit A, Ohmura K, et al. Critical roles for interleukin 1 and tumor necrosis factor alpha in antibody-induced arthritis. J Exp Med 2002;196:77 85.

40. Lamacchia C, Rodriguez E, Palmer G, et al. Articular in ammation is controlled by myeloid cell-derived interleukin 1 receptor antagonist during the acute phase of arthritis in mice. Ann Rheum Dis 2012;71:281 7.

41. Yazid S, Leoni G, Getting SJ, et al. Antiallergic cromones inhibit neutrophil recruitment onto vascular endothelium via annexin-A1 mobilization. Arterioscler Thromb Vasc Biol 2010:30:1718 24

42. Goulding NJ, Dixey J, Morand EF, et al. Differential distribution of annexins-I, -II, -IV, and $-\mathrm{VI}$ in synovium. Ann Rheum Dis 1995:54:841 5

43. Perretti M. The annexin 1 receptor(s): is the plot unravelling? Trends Pharmacol Sci 2003;24:574 9.

44. Dufton N, Hannon R, Brancaleone V, et al. Anti-in ammatory role of the murine formyl-peptide receptor 2: ligand-speci c effects on leukocyte responses and experimental in ammation. J Immunol 2010;184:2611 19.

45. Fischer $\mathbf{C P}$, Bode BP, Takahashi K, et al. Glucocorticoid-dependent induction of interleukin-6 receptor expression in human hepatocytes facilitates interleukin-6 stimulation of amino acid transport. Ann Surg 1996;223:610 18; discussion 61819
46. Granner DK. Restoration of sensitivity of cultured hepatoma cells to cyclic nucleotides shows permissive effect of dexamethasone. Nature 1976;259:572 3

47. Nigrovic PA, Binstadt BA, Monach PA, et al. Mast cells contribute to initiation of autoantibody-mediated arthritis via IL-1. Proc Natl Acad Sci USA 2007;104 232530.

48. Croxtall JD, van Hal PT, Choudhury $Q$, et al. Different glucocorticoids vary in their genomic and non-genomic mechanism of action in A549 cells. Br J Pharmacol 2002;135:511 19.

49. Vishwanath BS, Frey FJ, Bradbury M, et al. Adrenalectomy decreases lipocortin-l messenger ribonucleic acid and tissue protein content in rats. Endocrinology 1992;130:58591.

50. Solito E, de Coupade C, Parente L, et al. IL-6 stimulates annexin 1 expression and translocation and suggests a new biological role as class II acute phase protein. Cytokine 1998:10:514 21.

51. Tagoe CE, Marjanovic N, Park JY, et al. Annexin-1 mediates TNF-alpha-stimulated matrix metalloproteinase secretion from rheumatoid arthritis synovial broblasts. $\mathrm{J}$ Immunol 2008;181:281320.

52. D Acquisto F, Paschalidis N, Raza K, et al. Glucocorticoid treatment inhibits annexin-1 expression in rheumatoid arthritis CD4 + T cells. Rheumatology (Oxford) 2008;47:636 9.

53. Pimentel TA, Sampaio AL, D Acquisto F, et al. An essential role for mast cells as modulators of neutrophils in ux in collagen-induced arthritis in the mouse. Lab Invest 2011;91:33 42

54. Witko-Sarsat V, Reuter N, Mouthon L. Interaction of proteinase 3 with its associated partners: implications in the pathogenesis of Wegener $\mathrm{s}$ granulomatosis. Curr Opin Rheumatol 2010;22:1 7

55. Vong L, D Acquisto F, Pederzoli-Ribeil M, et al. Annexin 1 cleavage in activated neutrophils: a pivotal role for proteinase 3. J Biol Chem 2007;282:29998 30004.

56. Clark AR. Anti-in ammatory functions of glucocorticoid-induced genes. Mol Cell Endocrinol 2007:275:79 97.

57. Ayroldi E, Riccardi C. Glucocorticoid-induced leucine zipper (GILZ): a new important mediator of glucocorticoid action. FASEB J 2009:23:3649 58.

58. Abraham SM, Lawrence T, Kleiman A, et al. Antiin ammatory effects of dexamethasone are partly dependent on induction of dual speci city phosphatase 1. J Exp Med 2006;203:1883 9.

59. Beaulieu $\mathbf{E}$, Ngo D, Santos L, et al. Glucocorticoid-induced leucine zipper is an endogenous antiin ammatory mediator in arthritis. Arthritis Rheum 2010;62: 265161.

60. Solito $\mathbf{E}$, Christian HC, Festa M, et al. Post-translational modi cation plays an essential role in the translocation of annexin A1 from the cytoplasm to the cell surface. FASEB J 2006:20:1498 500.

61. Kirwan J. The origins, results and consequences of the 1995 Arthritis Research Campaign Low-Dose Glucocorticoid Study. Clin Exp Rheumatol 2011;29 (5 Suppl 68):S52 8. 